\title{
Global existence for a non-local mean curvature flow as a limit of a parabolic-elliptic phase transition model
}

\author{
D. HILHORST \\ Analyse Numérique et EDP, CNRS et Université de Paris-Sud, 91405 Orsay Cedex, France \\ E. LOGAK \\ Dpt de Mathématiques, Université de Cergy-Pontoise, 2 av. A. Chauvin, 95302 Cergy-Pontoise \\ Cedex, France \\ AND \\ R. SCHÄTZLE \\ Mathematisches Institut Albert-Ludwigs Universität, D79104 Freiburg, Germany \\ [Received 1 March 1999 and in revised form 23 February 2000]

\begin{abstract}
We consider a free boundary problem where the velocity depends on the mean curvature and on some non-local term. This problem arises as the singular limit of a reaction-diffusion system which describes the microphase separation of diblock copolymers. The interface may present singularities in finite time. This leads us to consider weak solutions on an arbitrary time interval and to prove the global-in-time convergence of solutions of the reaction-diffusion system.
\end{abstract}

\section{Introduction}

In this paper we consider the reaction-diffusion system

$$
P^{\epsilon} \begin{cases}u_{t}=\Delta u+\frac{1}{\epsilon^{2}}(f(u)-\epsilon v) & \text { in } \Omega \times \mathbb{R}_{+} \\ -\Delta v=u-f u & \text { in } \Omega \times \mathbb{R}_{+} \\ f v(., t)=0 & \text { for } t \geqslant 0 \\ u(x, 0)=g^{\epsilon}(x) & x \in \Omega,\end{cases}
$$

with periodic boundary conditions for $(u, v)$ on $\partial \Omega$, where

$$
\Omega=\left\{\left(x_{1}, \ldots, x_{N}\right) \in \mathbb{R}^{N}, \quad 0<x_{i}<\omega_{i}, \quad 1 \leqslant i \leqslant N\right\},
$$

with $\omega_{i}>0$ for $i=1,2, \ldots, N$. Here,

$$
f \phi=\frac{1}{|\Omega|} \int_{\Omega} \phi(x) \mathrm{d} x
$$

and $\epsilon>0$ is a small parameter. The nonlinear term $f(u)=-W^{\prime}(u)$ is the derivative of a doublewell potential with $W(-u)=W(u)$, having two minima at \pm 1 , with $W( \pm 1)=0$ and $W^{\prime \prime}( \pm 1)>0$. 
More precisely, $f$ is of bistable type and satisfies

$$
\begin{gathered}
f(-u)=-f(u), f(u)=0 \text { if and only if } u \in\{ \pm 1,0\}, \\
f^{\prime}( \pm 1)<0, \quad f^{\prime}(0)>0 .
\end{gathered}
$$

A typical example is given by $f(u)=u-u^{3}$. The initial condition $g^{\epsilon}$ is periodic of period $\omega=$ $\left(\omega_{1}, \omega_{2}, \ldots, \omega_{N}\right)$ and we will give the exact expression for $g^{\epsilon}$ in Section 2 below.

In what follows, we denote by $\left(u^{\epsilon}, v^{\epsilon}\right)$ the unique solution of Problem $\left(P^{\epsilon}\right)$ on $\Omega \times \mathbb{R}_{+}$which is periodic in space with period $\omega$. For $T>0$, we define $Q_{T}=\Omega \times(0, T)$. This problem arises as a model for microphase separation in diblock copolymers $[2,9,10]$. More precisely, $P^{\epsilon}$ is obtained as a gradient flow for the energy functional $F^{\epsilon}$ defined by

$$
F^{\epsilon}[u]=\int_{\Omega}\left[\frac{\epsilon|\nabla u|^{2}}{2}+\frac{W(u)}{\epsilon}+\frac{|\nabla v|^{2}}{2}\right],
$$

where the function $v$ is associated to the function $u$ by

$$
-\Delta v=u-f u \text { in } \Omega, \quad f v=0,
$$

together with periodic boundary conditions for $u$ and $v$. This functional is proposed in [10] and in [2] to describe the microphase separation of diblock copolymers where two different homopolymers are connected. This connectivity is responsible for long-range interactions between the two types of homopolymers which are represented by the non-local term $\int_{\Omega}|\nabla v|^{2}$ in $F^{\epsilon}$. Whereas minimizing the sum of the first two terms in the functional $F^{\epsilon}$ amounts to minimizing the area of the interface between the regions where $u \approx 1$ and $u \approx-1$, minimizing the last term involving $v$ tends to create oscillations in the function $u$, which in turn tends to increase the area of the interface. Indeed, in order to make the term $\int_{\Omega}|\nabla v|^{2}$ small enough, one has to let $u$ oscillate rapidly around $f u$. More precisely, if $u-f u$ converges weakly to 0 in $L^{2}(\Omega)$, then by compactness $\nabla v$ converges strongly to 0 in $L^{2}(\Omega)$. Thus we have to deal with two opposite tendencies.

It follows as in [8] that $P^{\epsilon}$ has a unique classical solution $\left(u^{\epsilon}, v^{\epsilon}\right)$. We are concerned in this paper with global-in-time convergence results about $\left(u^{\epsilon}, v^{\epsilon}\right)$. As for local-in-time results, it has been established in [8] that for suitable initial data $g^{\epsilon}$, the solution $\left(u^{\epsilon}, v^{\epsilon}\right)$ of system $P^{\epsilon}$ converges as $\epsilon \rightarrow 0$ to the solution $\left(u^{0}, v^{0}\right)$ of the free boundary problem (FBP) defined on $[0, T]$ by

$$
F B P \begin{cases}u^{0}(x, t)= \begin{cases}-1 & x \in \Omega_{t}^{-} \\ +1 & x \in \Omega_{t}^{+}\end{cases} \\ -\Delta v^{0}(x, t)=u^{0}(x, t)-f u^{0}(x, t) \mathrm{d} x & x \in \Omega_{t}^{-} \cup \Omega_{t}^{+} \\ f v^{0}(., t)=0 & x \in \Gamma_{t}=\Omega \backslash\left(\Omega_{t}^{-} \cup \Omega_{t}^{+}\right) \\ V_{n}=-(N-1) K+c_{0} v^{0}(x, t) & \\ \left.\Gamma_{t}\right|_{t=0}=\Gamma_{0}, & \end{cases}
$$

with periodic boundary data for $v^{0}$ on $\partial \Omega$. For each time $t \in[0, T]$, the free boundary $\Gamma_{t} \subset \subset \Omega$ is a smooth compact hypersurface without boundaries that divides $\Omega$ into two subdomains $\Omega_{t}^{-}$and $\Omega_{t}^{+}$, with $\Omega_{t}^{-} \subset \subset \Omega$. Here, $V_{n}$ denotes the normal velocity and $K$ the mean curvature on $\Gamma_{t}$. The 
constant $c_{0} \in \mathbb{R}$ only depends on $f$ and is related to the travelling wave solutions associated to the one-dimensional bistable equation. Well posedness for the FBP has been established in [4] and the associated local-in-time convergence result established in [8] reads as follows.

THEOREM 1.1 Let $\Gamma=\left(\left(\Gamma_{t} \times t\right)_{t \in[0, T]}, u^{0}, v^{0}\right)$ be a smooth solution to FBP on $[0, T]$. Then there exists a class of initial data $\left(g^{\epsilon}\right)_{0<\epsilon<1}$ such that if $\left(u^{\epsilon}, v^{\epsilon}\right)$ denotes the corresponding solution of $P^{\epsilon}$, we have

$$
\begin{aligned}
& \lim _{\epsilon \rightarrow 0} u^{\epsilon}(x, t)=u^{0}(x, t) \\
& \lim _{\epsilon \rightarrow 0} v^{\epsilon}(x, t)=v^{0}(x, t)
\end{aligned}
$$

for a.e. $(x, t) \in \Omega \times(0, T)$

Nevertheless even with smooth initial data $\Gamma^{0}$, the solution may develop a singularity in finite time. Thus a global-in-time convergence result needs to define some weak notion of solution of FBP.

Moreover, the inclusion of interfaces is not preserved in time for FBP (cf. [4]). This is why we cannot define a notion of viscosity solution for this problem. However, we will use the notion of viscosity solution for the interface motion equation in FBP with a fixed function $v^{0}$.

Let us mention other results about global-in-time convergence to viscosity solutions in similar phase transition systems. In [11], the authors consider a system where the evolution of $u^{\epsilon}$ is given by the same bistable equation as in $P^{\epsilon}$ but where $v^{\epsilon}$ is coupled to $u^{\epsilon}$ by a parabolic equation. This yields compactness in space-time for $v^{\epsilon}$. Our main contribution is to extend their approach to the case that the equation for $v^{\epsilon}$ is elliptic.

Note that we can prove similar global-in-time convergence results if the function pair $\left(u^{\epsilon}, v^{\epsilon}\right)$ satisfies an equation of the form

$$
u_{t}^{\epsilon}=\Delta u^{\epsilon}+\frac{1}{\epsilon^{2}}\left(f\left(u^{\epsilon}\right)-\epsilon v^{\epsilon}\right),
$$

with $\left(v^{\epsilon}\right)_{\epsilon>0}$ compact in $C^{0}\left(\bar{Q}_{T}\right)$. In particular, this is true in the case where $v^{\epsilon}=\int_{\Omega} u^{\epsilon}$, which has been studied in [5].

This paper is set out as follows. In Section 2, we first define our choice of initial data $g^{\epsilon}$ and then prove estimates for $\left(u^{\epsilon}, v^{\epsilon}\right)$ which imply the compactness of this sequence in $L^{1}\left(Q_{T}\right) \times C^{0}\left(\bar{Q}_{T}\right)$. The convergence result for a subsequence is then established in Section 3, using a related result by Barles et al. [3] in the case of a single equation. More precisely, we prove the following result.

THEOREM 1.2 For a suitable choice of initial data $g^{\epsilon}$, there exists a sequence $\epsilon_{j} \rightarrow 0$ such that for all $T>0$,

$$
\lim _{j \rightarrow+\infty} u^{\epsilon_{j}}=u \text { in } L^{1}\left(Q_{T}\right), \quad \lim _{j \rightarrow+\infty} v^{\epsilon_{j}}=v \text { in } C^{0}\left(\bar{Q}_{T}\right),
$$

for two functions $u$ and $v$ with $u= \pm 1$ a.e. in $Q_{T}$ and $v$ defined as the solution of

$$
-\Delta v=u-f u \text { a.e. in } Q_{T}, \quad f v=0 \text { on }[0, T],
$$

together with periodic boundary conditions. Moreover, if $\psi$ is the unique viscosity solution of the problem

$$
\left(P^{0}\right) \begin{cases}\frac{\partial \psi}{\partial t}-|\nabla \psi| \nabla \cdot\left(\frac{\nabla \psi}{|\nabla \psi|}\right)+c_{0}|\nabla \psi| v=0 & \text { on } \mathbb{R}^{N} \times \mathbb{R}_{+} \\ \psi(x, 0)=d^{0}(x) & \text { for } x \in \mathbb{R}^{N},\end{cases}
$$


for some positive constant $c_{0}$ wich only depends on $f$, the function $u$ satisfies the inequalities

$$
2 \chi_{\psi>0}-1 \leqslant u \leqslant 2 \chi_{\psi \geqslant 0}-1 \text { on } \mathbb{R}^{N} \times \mathbb{R}_{+} .
$$

\section{Estimates on the solutions of $P^{\epsilon}$}

In this section, we will consider the functions $\left(u^{\epsilon}, v^{\epsilon}\right)$ on $\Omega$. Thus the relation between $u^{\epsilon}$ and $v^{\epsilon}$ at any time $t \geqslant 0$ is

$$
\left\{\begin{array}{l}
-\Delta v^{\epsilon}(., t)=u^{\epsilon}(., t)-f u^{\epsilon}(., t) \quad \text { in } \Omega \\
\int_{\Omega} v^{\epsilon}(., t)=0
\end{array}\right.
$$

with $v^{\epsilon}$ satisfying periodic boundary conditions on $\partial \Omega$.

We first define our choice of initial data $g^{\epsilon}=\left.u^{\epsilon}\right|_{t=0}$.

In view of the assumptions (1.1) on $f=-W^{\prime}$, the equation in $u, f(u)-a=0$, has, for $|a| \leqslant \delta$ small enough, three solutions that we denote by $h_{-}(a)<h_{0}(a)<h_{+}(a)$, with $h_{ \pm}(0)= \pm 1$ and $h_{0}(0)=0$. Let us consider the travelling wave solution associated to the bistable non-linearity $f(u)-a$ which is defined as the unique solution $(c(a), q(r, a))$ of

$$
\begin{aligned}
& q_{r r}+c(a) q_{r}+f(q)-a=0 \\
& q( \pm \infty, a)=h_{ \pm}(a), \quad q(0, a)=\frac{h_{+}(a)+h_{-}(a)}{2} .
\end{aligned}
$$

It is standard that $(c, q)$ is uniquely determined and satisfies in particular for some constants $C>0$ and $\alpha>0$ and for all $r \in \mathbb{R}$ and $|a| \leqslant \delta$,

$$
\begin{aligned}
& 0 \leqslant q_{r} \leqslant C \mathrm{e}^{-\alpha|r|}, \quad-C \leqslant q_{a} \leqslant 0, \quad\left|q_{a r}\right| \leqslant C \\
& c(0)=0, \quad c^{\prime}(0)=c_{0}>0 \text { and } q(r, 0)=q_{0}(r) \text { satisfies } q_{0}^{\prime 2}=2 W\left(q_{0}\right) .
\end{aligned}
$$

Let $\Gamma^{0}=\partial \Omega_{0}^{-}$be a smooth given interface in $\Omega$, where $\Omega_{0}^{-} \subset \subset \Omega$ and $\Omega_{0}^{+}=\Omega \backslash \bar{\Omega}_{0}^{-}$are smooth subdomains of $\Omega$. Let $d^{0}(x)$ be the signed distance function to $\Gamma^{0}$ and let the function $u_{0}$ be defined in $\Omega$ by $u_{0}(x)= \pm 1$ for $x \in \Omega_{0}^{ \pm}$. We then consider the solution $v_{0}$ of

$$
-\Delta v_{0}=u_{0}-f u_{0} \text { in } \Omega, \quad f v_{0}=0,
$$

with $v_{0}$ satisfying periodic boundary conditions on $\partial \Omega$. The initial condition $g^{\epsilon}=\left.u^{\epsilon}\right|_{t=0}$ is then given by

$$
g^{\epsilon}(x)=q\left(\frac{d^{0}(x)}{\epsilon}, \epsilon v_{0}(x)\right),
$$

where $q$ is defined in (2.1). We now associate to $P^{\epsilon}$ the functional $E^{\epsilon}$ defined for $t \geqslant 0$ by

$$
E^{\epsilon}(t)=\int_{\Omega}\left[\frac{\epsilon\left|\nabla u^{\epsilon}\right|^{2}}{2}+\frac{W\left(u^{\epsilon}\right)}{\epsilon}+\frac{\left|\nabla v^{\epsilon}\right|^{2}}{2}\right] .
$$

Next we present some properties of the function $g^{\epsilon}$ and show in particular that the functional $E^{\epsilon}(0)$ is bounded. 
Lemma 2.1 The function $g^{\epsilon}$ defined in (2.5) satisfies for some constant $C>0$ and for all $0<\epsilon<$ $\epsilon_{0}$ small enough,

$$
\begin{aligned}
\text { (i) } & E^{\epsilon}(0) \leqslant C \\
\text { (ii) } & \left|g^{\epsilon}\right| \leqslant 1+C \epsilon \\
\text { (iii) } & \lim _{\epsilon \rightarrow 0} g^{\epsilon}(x)=u_{0}(x)= \pm 1 \text { for } x \in \Omega_{0}^{ \pm} .
\end{aligned}
$$

Proof. Properties (ii) and (iii) are straightforward using the properties of the travelling wave $q$. We now establish (i). First note that by (ii), the function $v^{\epsilon}(., 0)$ is uniformly bounded in $H^{1}(\Omega)$ and in $L^{\infty}(\Omega)$, so that the third term in $E^{\epsilon}(0)$ is bounded for all $\epsilon>0$. Moreover, using (2.2) and the definition of $g^{\epsilon}$, we have that

$$
g^{\epsilon}(x)=q_{0}\left(\frac{d^{0}(x)}{\epsilon}\right)+O(\epsilon)
$$

so that using (2.2) and (2.3), we get

$$
\begin{aligned}
\frac{W\left(g^{\epsilon}\right)}{\epsilon} & =\frac{W\left(q_{0}\left(\frac{d^{0}(x)}{\epsilon}\right)\right)}{\epsilon}+O(1) \\
& =\frac{1}{2 \epsilon}\left(q_{0}^{\prime}\right)^{2}\left(\frac{d^{0}(x)}{\epsilon}\right)+O(1) .
\end{aligned}
$$

As for the first term in $E^{\epsilon}(0)$, note that

$$
\nabla g^{\epsilon}=q_{r} \frac{\nabla d^{0}}{\epsilon}+q_{a} \epsilon \nabla v_{0}
$$

so that using again (2.2), we obtain

$$
\begin{aligned}
\epsilon\left|\nabla g^{\epsilon}\right|^{2} & =q_{r}^{2} \frac{\left|\nabla d^{0}\right|^{2}}{\epsilon}+O(\epsilon) \\
& =\left(q_{0}^{\prime}\right)^{2}\left(\frac{d^{0}(x)}{\epsilon}\right) \frac{\left|\nabla d^{0}\right|^{2}}{\epsilon}+O(1) .
\end{aligned}
$$

It then follows from (2.7), (2.9), and the fact that $\left|\nabla d^{0}\right|$ is bounded, for some constant $C>0$,

$$
E^{\epsilon}(0) \leqslant C \int_{\Omega} \frac{1}{\epsilon}\left(q_{0}^{\prime}\right)^{2}\left(\frac{d^{0}(x)}{\epsilon}\right) \mathrm{d} x+O(1) .
$$

Using the fact that $\left(q_{0}^{\prime}\right)$ decays exponentially fast to 0 at infinity and a parametrization $x \mapsto$ $\left(d^{0}, \mu\right) \in[-\delta,+\delta] \times \Gamma^{0}$ in a tubular neighbourhood of $\Gamma^{0}$, we deduce that for $\delta>0$ small 
enough,

$$
\begin{aligned}
\int_{\Omega} \frac{1}{\epsilon}\left(q_{0}^{\prime}\right)^{2}\left(\frac{d^{0}(x)}{\epsilon}\right) \mathrm{d} x & \leqslant \int_{\left|d^{0}\right| \leqslant \delta} \frac{1}{\epsilon}\left(q_{0}^{\prime}\right)^{2}\left(\frac{d^{0}(x)}{\epsilon}\right) \mathrm{d} x+O\left(\frac{\mathrm{e}^{-\alpha \frac{\delta}{\epsilon}}}{\epsilon}\right) \\
& \leqslant C \int_{\Gamma^{0}} \int_{-\delta}^{\delta} \frac{1}{\epsilon}\left(q_{0}^{\prime}\right)^{2}\left(\frac{r}{\epsilon}\right) \mathrm{d} r \mathrm{~d} \mu+O\left(\frac{\mathrm{e}^{-\alpha \frac{\delta}{\epsilon}}}{\epsilon}\right) \\
& \leqslant C \int_{-\frac{\delta}{\epsilon}}^{\frac{\delta}{\epsilon}}\left(q_{0}^{\prime}\right)^{2}(\sigma) \mathrm{d} \sigma+O\left(\frac{\mathrm{e}^{-\alpha \frac{\delta}{\epsilon}}}{\epsilon}\right) \\
& \leqslant C \int_{\mathbb{R}}\left(q_{0}^{\prime}\right)^{2}(\sigma) \mathrm{d} \sigma+C<+\infty,
\end{aligned}
$$

which finally yields (i). This completes the proof of Lemma 2.1.

We now prove estimates on $\left(u^{\epsilon}, v^{\epsilon}\right)$.

Lemma 2.2 There exists $\alpha \in(0,1)$ such that for all $C>0$ large enough and for $0<\epsilon<\epsilon_{0}$ small enough, if $g^{\epsilon}$ satisfies

$$
\left|g^{\epsilon}\right| \leqslant 1+C \epsilon \text { on } \Omega
$$

then the solution $\left(u^{\epsilon}, v^{\epsilon}\right)$ of $P^{\epsilon}$ satisfies

$$
-1-C \epsilon \leqslant u^{\epsilon}(t) \leqslant 1+C \epsilon \text { and }\left\|v^{\epsilon}(t)\right\|_{C^{1+\alpha}(\bar{\Omega})} \leqslant C_{1}
$$

for some positive constant $C_{1}$ and for all time $t \geqslant 0$.

Proof. First note that there exists a constant $C_{1}>0$ such that if $v$ satisfies

$$
\|-\Delta v\|_{L^{\infty}(\Omega)} \leqslant 2, \quad \int_{\Omega} v=0
$$

together with periodic boundary conditions, then by [1]

$$
\|v\|_{C^{1+\alpha}(\bar{\Omega})} \leqslant C_{1} .
$$

Moreover, we define

$$
C_{2}=\max _{s \in[-2,+2]}\left|f^{\prime \prime}(s)\right|,
$$

and we assume that

$$
\left|g^{\epsilon}\right| \leqslant 1+C \epsilon
$$

for some $C \geqslant \frac{C_{1}}{\left|f^{\prime}(1)\right|}$. We prove below that for all $C^{\prime}>C$ and for all time $t \geqslant 0$,

$$
\left|u^{\epsilon}(t)\right|<1+C^{\prime} \epsilon,
$$

if $0<\epsilon<\epsilon_{0}=\min \left(\frac{1}{C^{\prime}}, \frac{2\left(\left|f^{\prime}(1)\right| C^{\prime}-C_{1}\right)}{C_{2} C^{\prime 2}}\right)$. Assume by contradiction that there exists $\epsilon \in\left(0, \epsilon_{0}\right)$ and a first time $\tau=\tau(\epsilon)>0$ when this does not hold anymore. Note that for all $0 \leqslant t \leqslant \tau,\left|u^{\epsilon}(t)\right| \leqslant 2$ so that

$$
\left\|v^{\epsilon}(t)\right\|_{C^{1+\alpha}(\bar{\Omega})} \leqslant C_{1} .
$$


Defining $x_{\tau} \in \Omega$ as the point where $u^{\epsilon}(., \tau)$ achieves its maximum value, we deduce that

$$
u^{\epsilon}\left(x_{\tau}, \tau\right)=1+C^{\prime} \epsilon, \quad \frac{\partial u^{\epsilon}}{\partial t}\left(x_{\tau}, \tau\right) \geqslant 0, \quad \Delta u^{\epsilon}\left(x_{\tau}, \tau\right) \leqslant 0
$$

so that

$$
f\left(1+C^{\prime} \epsilon\right)-\epsilon v^{\epsilon}\left(x_{\tau}, \tau\right) \geqslant 0 .
$$

But, we have that

$$
\begin{aligned}
f\left(1+C^{\prime} \epsilon\right)-\epsilon v^{\epsilon}\left(x_{\tau}, \tau\right) & \leqslant f^{\prime}(1) C^{\prime} \epsilon+C_{2} C^{\prime 2} \frac{\epsilon^{2}}{2}+C_{1} \epsilon \\
& \leqslant \epsilon\left(-\left|f^{\prime}(1)\right| C^{\prime}+C_{1}+\frac{C_{2} C^{\prime 2} \epsilon}{2}\right)<0
\end{aligned}
$$

by the definitions of $C_{1}, C_{2}$ and $\epsilon_{0}$. This contradiction proves that $\left|u^{\epsilon}(t)\right| \leqslant 1+C \epsilon$ for all time $t \geqslant 0$.

Next we remark that $E^{\epsilon}$ is a Lyapunov functional for $P^{\epsilon}$. More precisely we have the following.

LEMMA 2.3 For all $t>0$,

$$
\frac{\mathrm{d} E^{\epsilon}(t)}{\mathrm{d} t}=-\epsilon \int_{\Omega}\left(u_{t}^{\epsilon}\right)^{2} \leqslant 0
$$

so that

$$
0 \leqslant E^{\epsilon}(t) \leqslant E^{\epsilon}(0) \leqslant C \text { and } \epsilon \int_{0}^{t} \int_{\Omega}\left(u_{t}^{\epsilon}\right)^{2} \leqslant C .
$$

Proof. Note that, using the fact that $f v^{\epsilon}=0$, the third term in $E^{\epsilon}$ can be rewritten as

$$
\int_{\Omega} \frac{\left|\nabla v^{\epsilon}\right|^{2}}{2}=\frac{1}{2} \int_{\Omega}\left(-\Delta v^{\epsilon}\right) v^{\epsilon}=\frac{1}{2} \int_{\Omega}\left(u^{\epsilon}-f u^{\epsilon}\right) v^{\epsilon}=\frac{1}{2} \int_{\Omega} u^{\epsilon} v^{\epsilon}
$$

so that

$$
\frac{\mathrm{d}}{\mathrm{d} t}\left(\int_{\Omega} \frac{\left|\nabla v^{\epsilon}\right|^{2}}{2}\right)=\frac{1}{2} \int_{\Omega} u_{t}^{\epsilon} v^{\epsilon}+\frac{1}{2} \int_{\Omega} u^{\epsilon} v_{t}^{\epsilon} .
$$

On the other hand,

$$
\frac{\mathrm{d}}{\mathrm{d} t}\left(\int_{\Omega} \frac{\left|\nabla v^{\epsilon}\right|^{2}}{2}\right)=\int_{\Omega}\left(-\Delta v^{\epsilon}\right) v_{t}^{\epsilon}=\int_{\Omega} u^{\epsilon} v_{t}^{\epsilon} .
$$

Combining the two above equalities yields

$$
\int_{\Omega} u^{\epsilon} v_{t}^{\epsilon}=\int_{\Omega} u_{t}^{\epsilon} v^{\epsilon}
$$

so that finally

$$
\frac{\mathrm{d}}{\mathrm{d} t}\left(\int_{\Omega} \frac{\left|\nabla v^{\epsilon}\right|^{2}}{2}\right)=\int_{\Omega} u_{t}^{\epsilon} v^{\epsilon} .
$$


It is then standard to differentiate $E^{\epsilon}$ and to get (2.10). The other inequalities in Lemma 2.3 are straightforward.

In order to deduce from Lemma 2.3 more precise estimates on $u^{\epsilon}$, we perform a change of functions. We define the auxiliary function $\tilde{u}^{\epsilon}=H\left(u^{\epsilon}\right)$, where

$$
H(s)=c \int_{0}^{s} \sqrt{W(\tau)} \mathrm{d} \tau
$$

with $c>0$ chosen such that $H( \pm 1)= \pm 1$. For instance, if $f(u)=2\left(u-u^{3}\right)$, then

$$
W(u)=\frac{\left(1-u^{2}\right)^{2}}{2} \text { and } H(s)= \begin{cases}\frac{1}{2}\left(3 s-s^{3}\right) & \text { for }|s| \leqslant 1 \\ 2 \operatorname{sgn}(s)+\frac{1}{2}\left(s^{3}-3 s\right) & \text { for }|s| \geqslant 1\end{cases}
$$

Let us now establish $B V$-type estimates on $\tilde{u}^{\epsilon}$.

LEMMA 2.4 There exists $C>0$ such that

$$
\int_{\Omega}\left|\nabla \tilde{u}^{\epsilon}\right| \leqslant C, \quad\left|\tilde{u}^{\epsilon}\right| \leqslant C
$$

and for all $0 \leqslant t_{1}<t_{2}$ we have

$$
\int_{t_{1}}^{t_{2}} \int_{\Omega}\left|\frac{\partial \tilde{u}^{\epsilon}}{\partial t}\right| \leqslant C\left|t_{2}-t_{1}\right|^{\frac{1}{2}}
$$

Proof. To begin with we remark that the boundedness of $\tilde{u}^{\epsilon}$ follows from the boundedness of $u^{\epsilon}$ established in Lemma 2.2. We have $\nabla \tilde{u}^{\epsilon}=H^{\prime}\left(u^{\epsilon}\right) \nabla u^{\epsilon}=c \sqrt{W\left(u^{\epsilon}\right)} \nabla u^{\epsilon}$ so that, using the Lyapunov functional, we get

$$
\int_{\Omega}\left|\nabla \tilde{u}^{\epsilon}\right| \leqslant C\left[\int_{\Omega} \epsilon\left|\nabla u^{\epsilon}\right|^{2}+\int_{\Omega} \frac{W\left(u^{\epsilon}\right)}{\epsilon}\right] \leqslant C
$$

for some $C>0$. Moreover, since $\frac{\partial \tilde{u}^{\epsilon}}{\partial t}=c \sqrt{W\left(u^{\epsilon}\right)} \frac{\partial u^{\epsilon}}{\partial t}$, we have that for all $0 \leqslant t_{1}<t_{2}$,

$$
\begin{gathered}
\int_{t_{1}}^{t_{2}} \int_{\Omega}\left|\frac{\partial \tilde{u}^{\epsilon}}{\partial t}\right| \\
\leqslant C\left|t_{2}-t_{1}\right|^{\frac{1}{2}}
\end{gathered}
$$

This ends the proof of Lemma 2.4

Next we prove that $\tilde{u}^{\epsilon}$ approximates $u^{\epsilon}$ in $L^{\infty}\left(0,+\infty ; L^{1}(\Omega)\right)$.

LEMMA 2.5 If we define

$$
\omega(\epsilon)=\sup _{t \geqslant 0}\left\|\tilde{u}^{\epsilon}(t)-u^{\epsilon}(t)\right\|_{L^{1}(\Omega)}
$$

then $\lim _{\epsilon \rightarrow 0} \omega(\epsilon)=0$. 
Proof. Note that both $u^{\epsilon}$ and $\tilde{u}^{\epsilon}$ are bounded so that, since $H$ is continuous, there exists $C>0$ such that for all $\delta>0$, we have

$$
\left|u^{\epsilon}-\tilde{u}^{\epsilon}\right| \leqslant C \chi_{\left\{|| u^{\epsilon}|-1| \geqslant \delta\right\}}+\max _{|| t|-1|<\delta}|t-H(t)|
$$

where $\chi_{A}$ is the characteristic function of the set $A$. Moreover, if $m[A]$ denotes the Lebesgue measure of the set $A$ in $\mathbb{R}^{N}$,

$$
\int_{\Omega} W\left(u^{\epsilon}\right) \geqslant \min _{|| t|-1| \geqslant \delta} W(t) m\left[|| u^{\epsilon}|-1| \geqslant \delta\right]
$$

so that by Lemma 2.3

$$
m\left[|| u^{\epsilon}|-1| \geqslant \delta\right] \leqslant C(\delta) \int_{\Omega} W\left(u^{\epsilon}\right) \leqslant \epsilon C(\delta)
$$

and, since $H( \pm 1)= \pm 1$,

$$
\max _{|| t|-1|<\delta}|t-H(t)|=\bar{\omega}(\delta) \rightarrow 0 \text { as } \delta \rightarrow 0 .
$$

Thus the integration of (2.11) in $\Omega$ yields

$$
\left\|u^{\epsilon}-\tilde{u}^{\epsilon}\right\|_{L^{1}(\Omega)} \leqslant \epsilon C(\delta)+C \bar{\omega}(\delta) .
$$

Choosing $\delta>0$ small enough and then $0<\epsilon<\epsilon(\delta)$ small enough, we deduce that $\left\|u^{\epsilon}-\tilde{u}^{\epsilon}\right\|_{L^{1}(\Omega)}$ tends to 0 as $\epsilon$ tends to 0 , uniformly with respect to $t \geqslant 0$. This completes the proof of Lemma 2.5.

We now derive uniform time estimates on $v^{\epsilon}$.

LEMmA 2.6 For all $\mu>0$, there exists $\delta>0$ such that for $\left|t_{2}-t_{1}\right|<\delta$, we have

$$
\left\|v^{\epsilon}\left(t_{2}\right)-v^{\epsilon}\left(t_{1}\right)\right\|_{C^{\alpha}(\bar{\Omega})}<\mu
$$

for all $\epsilon \in(0,1]$.

This inequality will be deduced from the two following lemmata.

Lemma 2.7 Let $C>0$ and $\alpha \in(0,1)$ be fixed. Then for all $\mu>0$, there exists $\tau>0$ such that if $v$ satisfies

$$
-\Delta v=u-f u \text { in } \Omega, \quad f v=0
$$

together with periodic boundary conditions and

$$
\|u\|_{L^{\infty}(\Omega)} \leqslant C \text { and }\|u\|_{L^{1}(\Omega)} \leqslant \tau,
$$

then $\|v\|_{C^{\alpha}(\bar{\Omega})} \leqslant \mu$. 
Proof. Assume by contradiction that the result does not hold. Then there exists $\mu>0$ and a sequence $\left\{u_{j}\right\}_{j \in \mathbb{N}}$ such that

$$
u_{j} \rightarrow 0 \text { in } L^{1}(\Omega) \text { and }\left\|u_{j}\right\|_{L^{\infty}(\Omega)} \leqslant C
$$

and such that if $v_{j}$ is the solution of

$$
-\Delta v_{j}=u_{j}-f u_{j} \text { in } \Omega, \quad f v_{j}=0
$$

together with periodic boundary conditions, then $\left\|v_{j}\right\|_{C^{\alpha}(\bar{\Omega})} \geqslant \mu>0$. By standard elliptic estimates and Sobolev imbeddings, it follows from (2.13) and (2.14) that $\left(v_{j}\right)_{j \in \mathbb{N}}$ is bounded in $W^{2, p}(\Omega)$ for all $p>1$ so that along a subsequence

$$
v_{j} \rightarrow v \text { in } C^{\alpha}(\bar{\Omega}) \text { and weakly in } W^{2, p}(\Omega) .
$$

Thus

$$
-\Delta v_{j}=u_{j}-f u_{j} \rightarrow 0=-\Delta v
$$

Therefore $v=0$, which is a contradiction. This ends the proof of Lemma 2.7.

We now establish time estimates for $u^{\epsilon}$.

LEMMA 2.8 For all $\tau>0$, there exists $\delta>0$ such that for $\left|t_{2}-t_{1}\right|<\delta$, we have

$$
\left\|u^{\epsilon}\left(t_{2}\right)-u^{\epsilon}\left(t_{1}\right)\right\|_{L^{1}(\Omega)}<\tau
$$

for all $\epsilon \in(0,1]$.

It follows from Lemma 2.5 and Lemma 2.4 that for all $0 \leqslant t_{1}<t_{2}$, we have

$$
\begin{aligned}
\left\|u^{\epsilon}\left(t_{2}\right)-u^{\epsilon}\left(t_{1}\right)\right\|_{L^{1}(\Omega)} \leqslant & \left\|u^{\epsilon}\left(t_{2}\right)-\tilde{u}^{\epsilon}\left(t_{2}\right)\right\|_{L^{1}(\Omega)}+\left\|\tilde{u}^{\epsilon}\left(t_{2}\right)-\tilde{u}^{\epsilon}\left(t_{1}\right)\right\|_{L^{1}(\Omega)} \\
& +\left\|\tilde{u}^{\epsilon}\left(t_{1}\right)-u^{\epsilon}\left(t_{1}\right)\right\|_{L^{1}(\Omega)} \\
\leqslant & 2 \omega(\epsilon)+\int_{t_{1}}^{t_{2}} \int_{\Omega}\left|\tilde{u}_{t}^{\epsilon}\right| \leqslant 2 \omega(\epsilon)+C \sqrt{t_{2}-t_{1}} .
\end{aligned}
$$

Also it follows from Lemma 2.3 that

$$
\begin{aligned}
\left\|u^{\epsilon}\left(t_{2}\right)-u^{\epsilon}\left(t_{1}\right)\right\|_{L^{1}(\Omega)} & \leqslant \int_{t_{1}}^{t_{2}} \int_{\Omega}\left|u_{t}^{\epsilon}\right| \leqslant\left(\int_{t_{1}}^{t_{2}} \int_{\Omega}\left(u_{t}^{\epsilon}\right)^{2}\right)^{\frac{1}{2}} \sqrt{t_{2}-t_{1}} \sqrt{|\Omega|} \\
& \leqslant \frac{C}{\sqrt{\epsilon}} \sqrt{t_{2}-t_{1}}
\end{aligned}
$$

Next we choose $\epsilon_{0}>0$ such that $\omega(\epsilon)<\frac{\tau}{4}$ for all $0<\epsilon<\epsilon_{0}$. Then it follows from (2.16) that for $\left|t_{2}-t_{1}\right|<\delta$

$$
\left\|u^{\epsilon}\left(t_{2}\right)-u^{\epsilon}\left(t_{1}\right)\right\|_{L^{1}(\Omega)} \leqslant \frac{\tau}{2}+C \sqrt{\delta}<\tau
$$

if $\delta \leqslant \epsilon_{0} \frac{\tau^{2}}{C^{2}}$. 
In the case that $\epsilon \geqslant \epsilon_{0}$, it follows from (2.17) that for $\left|t_{2}-t_{1}\right|<\delta$,

$$
\left\|u^{\epsilon}\left(t_{2}\right)-u^{\epsilon}\left(t_{1}\right)\right\|_{L^{1}(\Omega)} \leqslant C \sqrt{\frac{\delta}{\epsilon_{0}}}<\tau
$$

if $0<\delta<c_{0} \epsilon_{0} \tau^{2}$ is small enough. This completes the proof of Lemma 2.8.

ProOf OF Lemma 2.6 The inequality (2.12) follows from combining the conclusions of Lemma 2.7 and of Lemma 2.8. We are now in a position to establish the following convergence result.

Proposition 2.9 There exist two functions $(u, v)$ and a sequence $\epsilon_{j} \rightarrow 0$ such that

$$
\lim _{j \rightarrow+\infty} u^{\epsilon_{j}}=u \text { in } L^{1}\left(Q_{T}\right), \quad \lim _{j \rightarrow+\infty} v^{\epsilon_{j}}=v \text { in } C^{0}\left(\bar{Q}_{T}\right) .
$$

Moreover,

$$
u= \pm 1 \text { a.e. in } Q_{T} \text { and }-\Delta v=u-f u \text { a.e. in } Q_{T}, \quad f v=0 \text { on }[0, T]
$$

and $v$ satisfies periodic boundary conditions.

Proof. It follows from the estimates in Lemma 2.2 and Lemma 2.6 that the family $\left(v^{\epsilon}\right)_{\epsilon>0}$ is equicontinuous in $C^{0}\left(\bar{Q}_{T}\right)$ so that there exists a sequence $\epsilon_{j} \rightarrow 0$ and a function $v \in C^{0}\left(\bar{Q}_{T}\right)$ such that $v^{\epsilon_{j}} \rightarrow v$ uniformly on $\bar{Q}_{T}$. Moreover, it follows from Lemma 2.4 that $\left(\tilde{u}^{\epsilon}\right)_{\epsilon>0}$ is bounded in $B V\left(Q_{T}\right)$ so that by Rellich Lemma, there exists a function $u \in L^{1}\left(Q_{T}\right)$ such that $\tilde{u}^{\epsilon_{j}} \rightarrow u$ strongly in $L^{1}\left(Q_{T}\right)$. Since by Lemma 2.5

$$
\lim _{\epsilon \rightarrow 0} \sup _{t \geqslant 0}\left\|u^{\epsilon}(t)-\tilde{u}^{\epsilon}(t)\right\|_{L^{1}(\Omega)}=0,
$$

it follows that $u^{\epsilon_{j}} \rightarrow u$ strongly in $L^{1}\left(Q_{T}\right)$. Note that using the upper bound on the Lyapunov functional and Fatou's lemma, we again

$$
0 \leqslant \int_{\Omega} W(u) \leqslant \lim _{j \rightarrow+\infty} \int_{\Omega} W\left(u^{\epsilon_{j}}\right)=0,
$$

which implies that $W(u)=0$. Thus $u= \pm 1$ a.e. Finally, passing to the limit in the equation for $v^{\epsilon_{j}}$ yields that

$$
-\Delta v=u-f u \text { a.e. in } Q_{T} \text { and } f v=0 \text { on }[0, T]
$$

together with periodic boundary conditions. This ends the proof of Proposition 2.9.

REMARK 2.10 If $v_{0}(x)$ is the function defined in (2.4), we have that $v(x, 0)=v_{0}(x)$ for all $x \in \Omega$. In fact, it follows from the definition of $g^{\epsilon}$ in (2.5) that

$$
\lim _{\epsilon \rightarrow 0} g^{\epsilon}(x)=u_{0}(x) \text { for all } x \in \Omega
$$


so that passing to the limit in

$$
-\Delta v^{\epsilon_{j}}(x, 0)=g^{\epsilon_{j}}-f g^{\epsilon_{j}} \text { and } f v^{\epsilon_{j}}(x, 0)=0
$$

yields

$$
-\Delta v(x, 0)=u_{0}-f u_{0} \text { and } f v(x, 0)=0 .
$$

Thus $v(x, 0)=v_{0}(x)$.

\section{Convergence}

Let us consider the function $v$ obtained in the previous section as the limit of $v^{\epsilon_{n}}$ for some sequence $\epsilon_{n} \rightarrow 0$. Since $v$ is continuous on $\overline{Q_{T}}$, it follows from the results in [6] that there exists a unique continuous function $\psi(x, t)$ defined on $\mathbb{R}^{N} \times \mathbb{R}_{+}$which is the viscosity solution of

$$
\left(P^{0}\right) \begin{cases}\frac{\partial \psi}{\partial t}-|\nabla \psi| \nabla \cdot\left(\frac{\nabla \psi}{|\nabla \psi|}\right)+c_{0}|\nabla \psi| v=0 & \text { on } \mathbb{R}^{N} \times \mathbb{R}_{+} \\ \psi(x, 0)=d^{0}(x) & \text { for } x \in \mathbb{R}^{N},\end{cases}
$$

where $c_{0}>0$ is defined in (2.3).

\subsection{Viscosity solutions}

We now recall the definition of the viscosity solution of Problem $\left(P^{0}\right)$. Let us define for any symmetric $(n, n)$ matrix $X \in S_{n}$ and for any vector $p \in \mathbb{R}^{n} \backslash\{0\}$

$$
K(p, X)=-\operatorname{Tr}(X)+\frac{(X p, p)}{|p|^{2}}
$$

and

$$
F(x, t, p, X)=K(p, X)+c_{0}|p| v(x, t)
$$

so that Problem $\left(P^{0}\right)$ can be rewritten as

$$
\left(P^{0}\right) \begin{cases}\frac{\partial \psi}{\partial t}+F\left(x, t, \nabla \psi, D^{2} \psi\right)=0 & \text { on } \mathbb{R}^{N} \times \mathbb{R}_{+} \\ \psi(x, 0)=d^{0}(x) & \text { for } x \in \mathbb{R}^{N} .\end{cases}
$$

We denote by $K^{*}\left(K_{*}\right)$ the upper semi-continuous (lower semi-continuous) envelope of $K$. For instance, we recall that

$$
K^{*}(p, X)=\limsup _{\epsilon \rightarrow 0}\{K(q, Y),|p-q|<\epsilon,|X-Y|<\epsilon\} .
$$

It can be shown [7] that

$$
K_{*}(p, X)=-\sum_{i=2}^{n} \lambda_{i}(X) \text { and } K^{*}(p, X)=-\sum_{i=1}^{n-1} \lambda_{i}(X),
$$


where $\lambda_{1}(X) \leqslant \lambda_{2}(X) \leqslant \ldots \leqslant \lambda_{n}(X)$ are the eigenvalues of $X$. We then define the upper semicontinuous (lower semi-continuous) envelope of $F$ by

$$
F^{*}(x, t, p, X)=K^{*}(p, X)+c_{0}|p| v(x, t)
$$

and

$$
F_{*}(x, t, p, X)=K_{*}(p, X)+c_{0}|p| v(x, t)
$$

respectively.

Definition 3.1 A function $u: R^{N} \times(0, T) \rightarrow R$ is called a viscosity subsolution (respectively. supersolution) of the equation

$$
u_{t}+F\left(x, t, u, \nabla u, D^{2} u\right)=0,(x, t) \in R^{N} \times(0, T)
$$

(which we formally write as

$$
u_{t}+F\left(x, t, u, \nabla u, D^{2} u\right) \leqslant 0,(x, t) \in R^{N} \times(0, T)(\geqslant 0 \text { for the supersolution) })
$$

if $u$ is upper semi-continuous on $R^{N} \times[0, T]$ (lower semi-continuous on $R^{N} \times[0, T]$ for the supersolution) and if

$$
\begin{aligned}
& \phi_{t}\left(x_{0}, t_{0}\right)+F_{*}\left(x_{0}, t_{0}, u\left(x_{0}, t_{0}\right), \nabla \phi\left(x_{0}, t_{0}\right), D^{2} \phi\left(x_{0}, t_{0}\right)\right) \leqslant 0 \\
& \left(\phi_{t}\left(x_{0}, t_{0}\right)+F^{*}\left(x_{0}, t_{0}, u\left(x_{0}, t_{0}\right), \nabla \phi\left(x_{0}, t_{0}\right), D^{2} \phi\left(x_{0}, t_{0}\right)\right) \geqslant 0 \text { for the supersolution }\right)
\end{aligned}
$$

for all $\phi$ in $C^{2,1}\left(R^{N} \times[0, T]\right)$ and all local maxima (local minima) $\left(x_{0}, t_{0}\right)$ of the function $(u-\phi)$. The function $u$ is a viscosity solution of (3.3) if it is both a sub- and a supersolution.

The last part of this paper is devoted to the proof of the following proposition

Proposition 3.2 Let $(u, v)$ be the limit of $\left(u^{\epsilon_{n}}, v^{\epsilon_{n}}\right)$ for some sequence $\epsilon_{n} \rightarrow 0$ given by Proposition 2.9. Let $\psi(x, t)$ be the unique viscosity solution of Problem $\left(P^{0}\right)$ associated to the function $v$. Then,

$$
2 \chi_{\psi>0}-1 \leqslant u \leqslant 2 \chi_{\psi \geqslant 0}-1 \text { on } \mathbb{R}^{N} \times \mathbb{R}_{+} .
$$

Equivalently, $\lim _{n \rightarrow+\infty} u^{\epsilon_{n}}= \pm 1$ a.e. with

$$
\lim _{n \rightarrow+\infty} u^{\epsilon_{n}}(x, t)=+1 \text { if } \psi(x, t)>0 \text { and } \lim _{n \rightarrow+\infty} u^{\epsilon_{n}}(x, t)=-1 \text { if } \psi(x, t)<0 .
$$

Let $\tilde{v} \in C^{0}\left(\bar{Q}_{T}\right)$ be arbitrary and consider the unique viscosity solution $\tilde{\psi}$ of Problem $\left(P_{\tilde{v}}^{0}\right)$,

$$
\left(P_{\tilde{v}}^{0}\right) \begin{cases}\frac{\partial \tilde{\psi}}{\partial t}-|\nabla \tilde{\psi}| \nabla \cdot\left(\frac{\nabla \tilde{\psi}}{\mid \nabla \tilde{\psi})}\right)+c_{0}|\nabla \tilde{\psi}| \tilde{v}=0 & \text { on } \mathbb{R}^{N} \times \mathbb{R}_{+} \\ \tilde{\psi}(x, 0)=d^{0}(x) & \text { for } x \in \mathbb{R}^{N} .\end{cases}
$$

We now establish two propositions about solutions of Problem $\left(P_{\tilde{v}}^{0}\right)$ which rely on properties established by [6].

Proposition 3.3 Let $\tilde{v}_{1} \leqslant \tilde{v}_{2}$ and let $\tilde{\psi}_{1}$ and $\tilde{\psi}_{2}$ be the solutions of Problems $\left(P_{\tilde{v}_{1}}^{0}\right)$ and $\left(P_{\tilde{v}_{2}}^{0}\right)$, respectively. Then $\tilde{\psi}_{1} \geqslant \tilde{\psi}_{2}$ on $\mathbb{R}^{N} \times \mathbb{R}_{+}$. 
Proof. Let $\tilde{\psi}_{1}\left(\tilde{\psi}_{2}\right)$ be the viscosity solution of Problem $\left(P_{\tilde{v}_{1}}^{0}\right)\left(\left(P_{\tilde{v}_{2}}^{0}\right)\right)$. We show that $\tilde{\psi}_{1}$ is a viscosity supersolution of Problem $\left(P_{\tilde{v}_{2}}^{0}\right)$, which implies the above result by the standard comparison principle for viscosity solutions [6]. Next we write that $\tilde{\psi}_{1}$ is a viscosity supersolution of Problem $\left(P_{\tilde{v}_{1}}^{0}\right)$. For any test function $\phi$ such that $\left(\tilde{\psi}_{1}-\phi\right)$ has a local minimum at $\left(x_{0}, t_{0}\right)$, we have that

$$
\phi_{t}\left(x_{0}, t_{0}\right)+K^{*}\left(\nabla \phi\left(x_{0}, t_{0}\right), D^{2} \phi\left(x_{0}, t_{0}\right)\right)+c_{0}\left|\nabla \phi\left(x_{0}, t_{0}\right)\right| \tilde{v}_{1}\left(x_{0}, t_{0}\right) \geqslant 0,
$$

which implies that

$$
\phi_{t}\left(x_{0}, t_{0}\right)+K^{*}\left(\nabla \phi\left(x_{0}, t_{0}\right), D^{2} \phi\left(x_{0}, t_{0}\right)\right)+c_{0}\left|\nabla \phi\left(x_{0}, t_{0}\right)\right| \tilde{v}_{2}\left(x_{0}, t_{0}\right) \geqslant 0,
$$

which yields the result.

Proposition 3.4 We define $\tilde{v}_{\alpha}=\tilde{v}+\alpha$ for $\alpha \in \mathbb{R}$. Let $\tilde{\psi}_{\alpha}(\tilde{\psi})$ be the solution of Problem $\left(P_{\tilde{v}_{\alpha}}^{0}\right)$ $\left(\left(P_{\tilde{v}}^{0}\right)\right)$. Then $\lim _{\alpha \rightarrow 0} \tilde{\psi}_{\alpha}=\tilde{\psi}$ and the convergence is uniform on compact sets of $\mathbb{R}^{N} \times \mathbb{R}_{+}$.

Proof. We define

$$
\begin{aligned}
\psi^{+}(x, t)= & \lim _{\nu \rightarrow 0} \sup \left\{\tilde{\psi}_{\alpha}(z, \theta), \text { for all } 0 \leqslant \alpha \leqslant v\right. \text { and for all } \\
& \left.(z, \theta) \in \mathbb{R}^{N} \times(0, T) \text { such that }|x-z| \leqslant v \text { and }|t-\theta| \leqslant v\right\}
\end{aligned}
$$

and

$$
\begin{aligned}
\psi^{-}(x, t)= & \lim _{\nu \rightarrow 0} \inf \left\{\tilde{\psi}_{\alpha}(z, \theta), \text { for all } 0 \leqslant \alpha \leqslant \nu\right. \text { and for all } \\
& \left.(z, \theta) \in \mathbb{R}^{N} \times(0, T) \text { such that }|x-z| \leqslant v \text { and }|t-\theta| \leqslant \nu\right\} .
\end{aligned}
$$

One can show [7] that

$$
\psi^{+}(x, 0)=\psi^{-}(x, 0)=d^{0}(x) \text { on } \mathbb{R}^{N} .
$$

Note that $\psi^{-} \leqslant \psi^{+}$. The reverse inequality is a consequence of the comparison principle and of the following lemma, which one can prove as in [7].

Lemma $3.5 \psi^{+}\left(\psi^{-}\right)$is a subsolution (supersolution) to Problem $\left(P_{\tilde{v}}^{0}\right)$.

Finally let us recall a result by Barles et al. [3] about the perturbed Allen-Cahn equation corresponding to $\tilde{v}$,

$$
u_{t}=\Delta u+\frac{1}{\epsilon^{2}}(f(u)-\epsilon \tilde{v}),
$$

where $\tilde{v}=\tilde{v}(x, t)$ is a smooth function. The result in [3] then reads as follows.

Proposition 3.6 Let $\Gamma^{0} \subset \mathbb{R}^{N}$ be a closed set and let $d\left(x, \Gamma^{0}\right)$ be the signed distance function to $\Gamma^{0}$. We define

$$
\tilde{g}^{\epsilon}(x)=q\left(\frac{d\left(x, \Gamma^{0}\right)}{\epsilon}, \epsilon \tilde{v}(x, 0)\right),
$$

where $q(., a)$ is defined in (2.1). Let $\tilde{v} \in C^{2,1}\left(\mathbb{R}^{N} \times \mathbb{R}_{+}\right)$be fixed. Let $\left(P_{\tilde{v}}^{\epsilon}\right)$ denote the Allen-Cahn equation with right-hand-side $\tilde{v}$,

$$
\left(P_{\tilde{v}}^{\epsilon}\right) \begin{cases}u_{t}=\Delta u+\frac{1}{\epsilon^{2}}(f(u)-\epsilon \tilde{v}) & \text { in } \mathbb{R}^{N} \times \mathbb{R}_{+} \\ u(x, 0)=\tilde{g}^{\epsilon}(x) & x \in \mathbb{R}^{N}\end{cases}
$$


and let $\tilde{u}^{\epsilon}$ be the unique solution of this problem. Let $\tilde{\psi}(x, t)$ be the solution of Problem $\left(P_{\tilde{v}}^{0}\right)$ with $\tilde{\psi}(x, 0)=d\left(x, \Gamma^{0}\right)$. Then, for all $(x, t) \in \mathbb{R}^{N} \times \mathbb{R}_{+}$,

$$
\lim _{\epsilon \rightarrow 0} \tilde{u}^{\epsilon}(x, t)=+1 \text { if } \tilde{\psi}(x, t)>0 \text { and } \lim _{\epsilon \rightarrow 0} \tilde{u}^{\epsilon}(x, t)=-1 \text { if } \tilde{\psi}(x, t)<0 .
$$

We now come to the proof of the convergence result.

Proof of Proposition 3.2 Let $\left(x_{0}, t_{0}\right) \in \mathbb{R}^{N} \times \mathbb{R}_{+}$be fixed and assume that $\psi\left(x_{0}, t_{0}\right)>0$. For any $\alpha>0$, since $v$ is continuous on $\bar{Q}_{T}$, there exists a smooth function $\tilde{v}$ which depends on $\alpha$ such that

$$
v+2 \alpha \geqslant \tilde{v} \geqslant v+\alpha \text { on } \bar{Q}_{T} .
$$

Using Proposition 3.4, we can choose $\alpha>0$ small enough so that $\tilde{\psi}\left(x_{0}, t_{0}\right)>0$, where $\tilde{\psi}$ is the solution of Problem $\left(P_{\tilde{v}}\right)$. We will compare the function $u^{\epsilon}$ associated to Problem $\left(P^{\epsilon}\right)$ to the solution of Problem $\left(P_{\tilde{v}}^{\epsilon}\right)$. First note that since $q_{a} \leqslant 0$, we have that, since $v(x, 0)=v^{0}(x) \leqslant$ $\tilde{v}(x, 0)$,

$$
g^{\epsilon}(x, 0)=q\left(\frac{d\left(x, \Gamma^{0}\right)}{\epsilon}, \epsilon v^{0}(x)\right) \geqslant \tilde{g}^{\epsilon}(x) .
$$

Moreover, for $n \in \mathbb{N}$ large enough, we have that $v^{\epsilon_{n}} \leqslant v+\alpha \leqslant \tilde{v}$ on $\bar{Q}_{T}$ so that in view of Lemma 2.2, of inequality (3.4), and of the standard comparison principle, we have that

$$
\tilde{u}^{\epsilon_{n}} \leqslant u^{\epsilon_{n}} \leqslant 1+C \epsilon_{n} \text { on } \bar{Q}_{T},
$$

where $\tilde{u}^{\epsilon_{n}}$ is the solution of Problem $\left(P_{\tilde{v}}^{\epsilon_{n}}\right)$ defined above. Using Proposition 3.6, we deduce that

$$
\lim _{n \rightarrow+\infty} \tilde{u}^{\epsilon_{n}}\left(x_{0}, t_{0}\right)=1
$$

which implies by (3.5) that

$$
u\left(x_{0}, t_{0}\right)=\lim _{n \rightarrow+\infty} u^{\epsilon_{n}}\left(x_{0}, t_{0}\right)=1 .
$$

Similar arguments show that if $\psi\left(x_{0}, t_{0}\right)<0$, then $u\left(x_{0}, t_{0}\right)=-1$.

\section{Acknowledgement}

The authors wish to acknowledge the support of the TMR project Nonlinear Parabolic Partial Differential Equations: Methods and Applications ERB FMRX CT98 0201.

\section{REFERENCES}

1. Agmon, S., Douglis, A., \& Nirenberg, L. Estimates near the boundary for solutions of elliptic partial differential equations satisfying general boundary conditions I. Comm. Pure Appl. Math. 12, (1959) 623-727.

2. Bahiana, M. \& Oono, Y. Cell dynamical system approach to block copolymers. Phys. Rev. A 41, (1990) 6763-6771.

3. Barles, G., Soner, H. M., \& Souganidis, P. E. Front propagation and phase field theory. SIAM J. Cont. Opt. 31, (1993) 439-469. 
4. Bonami, A., Hilhorst, D., \& Logak, E. Modified motion by mean curvature: local existence and uniqueness and qualitative properties. Differential and Integral Equations to appear.

5. Chen, X., Hilhorst, D., \& LogAK, E. Asymptotic behaviour of an Allen-Cahn equation with a nonlocal term. Nonlinear Analysis TMA 28(7), (1997) 1283-1298.

6. Giga, Y., Goto, S., IshiI, H., \& SATO, M. Comparison principle and convexity preserving properties for singular degenerate parabolic equations on unbounded domains. Indiana Univ. Math. J. 40(2), (1991) 443-470.

7. Henry, M., Hilhorst, D., \& SchÄtzle, R. Convergence to a viscosity solution for an advectionreaction-diffusion equation arising from a chemotaxis-growth model. Hiroshima Math. Journal 29, (1999) 591-630.

8. LOGAK, E. Singular limit of reaction-diffusion systems and modified motion by mean curvature. In Proceedings of the Royal Soc. of Edinburgh. to appear.

9. Nishiura, Y. \& OHnishi, I. Some mathematical aspects of the micro-phase separation in diblock copolymers. Physica D 84, (1995) 31-39.

10. ОНTA, T. \& KAWASAKI, K. Equilibrium morphology of block copolymer melts. Macromolecules 19, (1986) 2621-2632.

11. Souganidis, P. E. \& Soravia, P. Phase field theory for Fitzhugh-Nagumo type systems. SIAM J. Math. Anal. 27, (1996) 1341-1359. 